\title{
Stochastic Properties of Colliding Hard Spheres in a Non-equilibrium Thermal Bath
}

\author{
Armando Bazzani, Silvia Vitali, Carlo E. Montanari, Matteo Monti, \\ Sandro Rambaldi, and Gastone Castellani
}

\begin{abstract}
We consider the problem of describing the dynamics of a test particle moving in a thermal bath using the stochastic differential equations. We briefly recall the stochastic approach to the Brownian based on the statistical properties of collision theory for a gas of elastic particles and the molecular chaos hypothesis. The mathematical formulation of the Brownian motion leads to the formulation of the Ornstein-Uhlenbeck equation that provides a stationary solution consistent with the Maxwell-Boltzmann distribution. According to the stochastic thermodynamics, we assume that the stochastic differential equations allow to describe the transient states of the test particle dynamics in a thermal bath and it extends their application to the study of the non-equilibrium statistical physics. Then we consider the problem of the dynamics of a test massive particle in a non homogeneous thermal bath where a gradient of temperature is present. We discuss as the existence of a local thermodynamics equilibrium is consistent with a Stratonovich interpretation of the stochastic differential equations with a multiplicative noise. The stochastic model applied to the test particle dynamics implies the existence of a long transient state during which the particle shows a net drift toward the cold region of the system. This effect recalls the thermophoresis phenomenon performed by large molecule in a solution in response to a macroscopic temperature gradient and it can be explained as an effect of the non-locality character of the collision interactions between the test particle and the thermal bath particles. To validate the stochastic model assumptions we analyze
\end{abstract}

A. Bazzani $(\varangle) \cdot$ C. E. Montanari · S. Rambaldi

Department DIFA, University of Bologna, INFN sezione di Bologna via Irnerio 46, 40126

Bologna, Italy

e-mail: armando.bazzani@unibo.it

S. Vitali

Basque Center for Applied Mathematics, Alameda de Mazarredo 14, 48009

Bilbao, Bizkaia, Spain

M. Monti

EPFL IC École Polytechnique Fédérale de Lausanne, Station 14, 1015 LausanneLausanne, Switzerland

G. Castellani

Department DIMES, University of Bologna, INFN sezione di Bologna,

Via Giuseppe Massarenti, 1, 0138 Bologna, Italy

(C) The Author(s), under exclusive license to Springer Nature Switzerland AG 2021

L. Beghin et al. (eds.), Nonlocal and Fractional Operators, SEMA SIMAI Springer

Series 26, https://doi.org/10.1007/978-3-030-69236-0_3 
the simulation results of the 2-dimensional hard sphere gas obtained by using an event-based computer code, that solves exactly the sphere dynamics. The temperature gradient is simulated by the presence of two reflecting boundary conditions at different temperature. The simulations suggest that existence of a local thermodynamic equilibrium is justified and highlight the presence of a drift in the average dynamics of an ensemble of massive particles. The results of the paper could be relevant for the applications of stochastic dynamical systems to the non-equilibrium statistical physics that is a key issue for the Complex Systems Physics.

Keywords Collisional theory $\cdot$ Stochastic differential equations • Non-equilibrium stationary states

\section{Introduction}

The understanding of the non-equilibrium statistical systems is one of the main issue of Complex Systems Physics. Modeling biological, biochemical or socio-economical complex systems usually copes with the problem of describing the evolution of a system out of equilibrium [1]. Even if one restricts the study to consider nonequilibrium stationary states (NESS) [2], the existence of universal laws cope with the peculiarities of each statistical system out of equilibrium, where some details of the interactions among elementary components play a relevant role. Despite of a great effort to find a general theoretical approach to non-equilibrium thermodynamics, the scientific community is still discussing if there exists an analogous of the Entropy Principle [3] that governs the relaxation process toward the equilibrium state. In this framework the stochastic differential equations have been recognized a powerful tool to study the dynamics of statistical systems [4]. Even if these equations cannot be derived from the fundamental physical laws, the universality of the Central Limit Theorems (CTL) and the chaotic properties of many degrees of freedom dynamical systems pointed out by Ergodic Theory, justify the applications to real systems. Some relations or assumptions that are at the base of the stochastic model approach, as the fluctuation-dissipation relations [5] or the reversibility properties of the stationary solution (Onsager relations [6]) could be extended to the NESS states. In this work we consider the problem of explaining the statistical properties of a gas of colliding elastic particles by means of the stochastic differential equations. The assumption of the molecular chaos allows to describe the dynamics of a massive particle in the gas as a Brownian motion in the vanishing mass limit for the gas particles and infinitely frequent collisions. We discuss the possible extension of this approach to consider a NESS state of a hard sphere gas between two reflecting boundary conditions at different temperatures. Assuming that the gas realizes a local pressure equilibrium, we derive some scaling laws for the relevant parameters that define the collision theory. The particle dynamics is described by a stochastic differential equation with a multiplicative noise, due to the dependence of the temperature from the position. Our result is that for a finite particle mass and a finite collision frequency, 
one has to interpret the stochastic differential equation according to Stratonovich [7] to take into account the correlation among successive collisions in order to get the local equilibrium of pressure in the NESS state. Under this point of view, the collision dynamics is non-local and one has to introduce an effective force to take into account the effect of temperature gradient. We extend this result to describe the dynamics of a massive test particle in the non-uniform thermal bath and we show that the model implies a long correlation time in the evolution, that induce an average net drift of the particle toward the colder regions of the thermal bath. The effect increases as one increases the mass of the test particle and it may recall the thermophoresis phenomenon observed when real particles fluctuate in presence of a temperature gradient [8]. In order to get a validation of the proposed approach, we have performed numerical simulations for a 2-dimensional hard sphere gas. The simulation code [9] uses an event based algorithm, that allows an exact (with the round-off errors of the double precision) integration of the collision dynamics of $10^{4} \div 10^{5}$ elastic particles. Such numbers are suitable to simulate a condition of moderate density where the particles may explore all the available space (i.e. we are simulating a gaseous state), but they relax to a local equilibrium state justified by the application of a Central Limit Theorem (CLT). The simulation results suggest that the stochastic differential equations are indeed able to explain some statistical features of the collision dynamics in presence of a temperature gradient. Our results are consistent with the Stochastic Thermodynamics approach to non-equilibrium statistical physics [10]. However a quantitative relation among the thermophoresis phenomenon in chemistry, the relaxation process we observe in the simulation of an ensemble of massive test particles and the solution of the stochastic differential equation, requires further studies.

The paper is organized as follows: in the second section we briefly present the fundamental concepts of collision theory that justify a stochastic model for the Brownian motion and its possible extension to consider the effect of a temperature gradient in the thermal bath; in the third section we discuss the results of molecular dynamics simulations and the possible validation of the stochastic model description both in a equilibrium and in a NESS state; finally some conclusions and perspective are outlined.

\section{Collision Theory and Stochastic Differential Equation}

To understand the mesoscopic description of statistical systems using the stochastic dynamical systems theory, we study the collisions among particles in the limit of local, instantaneous and binary interactions. When it is possible to neglect the details of the microscopic dynamics using the CLT, the statistical properties are reproduced by mesoscopic models, where one only considers the statistical effects of fluctuations. A formal approach starts from some physical assumptions on the microscopic dynamics: 
1. we distinguish three different time scales: the interaction time scale $\Delta \tau$ (i.e. the time duration of a collision that we assume very small), the collision scale time $\tau$ that measures the elapsed time between successive collisions (i.e. $\tau$ is the average correlation time of microscopic dynamics and $\tau^{-1}$ the collisions frequency) and $\Delta t \gg \tau$ the evolution time scale of the dynamical variables;

2. we assume the molecular chaos: i.e. the collisions are instantaneous and successive collisions can be considered independent events (we have a discontinuous dynamics in the momentum space of a test particle);

3. the collisions are binary: we do not consider multiple collisions or collective mean field effects;

4. we have conservation laws in the collision dynamics: we consider elastic collisions where kinetic energy and momentum are preserved.

The application of the CLT to the momentum dynamics justifies a stochastic approach to model the elastic collisions effect on a test particle of mass $M$ in a thermal bath simulated by an ensemble of particles with a mass $m \ll M$. If $\tau^{-1}$ is the collision frequency (i.e. $\tau$ is the average time interval between successive collisions), in the limit $m \rightarrow 0$ and $\tau \rightarrow 0$ with the ratio $m / \tau$ kept constant (the Brownian motion limit), the evolution of the momentum $\boldsymbol{P}(\boldsymbol{t})$ is described by the stochastic dynamical system

$$
\boldsymbol{P}(t+\Delta t)=\boldsymbol{P}(\boldsymbol{t})-\frac{\gamma}{M} \boldsymbol{P}(\boldsymbol{t}) \Delta t+\sqrt{2 \gamma \Delta t T} \boldsymbol{\xi}(t)
$$

where $T$ is the temperature of the thermal bath according to the expectation value over the gas particles

$$
E\left(\frac{p^{2}}{2 m}\right)=\frac{3}{2} T
$$

and the parameter $\gamma$

$$
\gamma=\frac{2 m}{\tau} E\left(\sin ^{2} \frac{\theta}{2}\right)
$$

contains the statistical information of the collision dynamics through the expectation value $E\left(\sin ^{2} \theta / 2\right)$ ( $\theta$ is the deflection angle due to an elastic collision and the average value is computed over all the possible collisions of the test particle with the gas particles). We observe that the Einstein fluctuation-dissipation relation is satisfied. The time step $\Delta t$ is the evolution time scale for the momentum $\boldsymbol{P}(t)$ (i.e. $\boldsymbol{P}(t)$ can be considered constant during $\Delta t$ with an error that vanishes in the Brownian motion limit) and $\boldsymbol{\xi}(k \Delta t) k \in \mathbb{N}^{0}$ are independent standard Gaussian variables. The justification of the stochastic dynamics (1) requires that the collision dynamics could be described as the sum of independent events and that the gas particles colliding with the test particle are 'thermalized' so that the expectation value of the kinetic energy is $3 / 2 T$ (the Boltzmann constant is set to one). The last requirement is a key point since it means that the test particle can be considered in a local thermal equilibrium at each time interval $\Delta t$. In the limit $\Delta t \rightarrow 0$ the random walk (1) realizes the Brownian motion for the test particle 


$$
\begin{aligned}
& d \boldsymbol{P}=-\frac{\gamma}{M} \boldsymbol{P} d t+\sqrt{2 \gamma T} d \boldsymbol{w}(t) \\
& d \boldsymbol{X}=\frac{\boldsymbol{P}}{M} d t
\end{aligned}
$$

where $\boldsymbol{w}(t)$ is a vector Wiener process. To compare the analytical approach with numerical results, we consider a 2-dimensional hard spheres gas in the sequel: in such a case one explicitly evaluates $E\left(\sin ^{2} \theta / 2\right)=1 / 3$ [11]. The Brownian motion limit requires that the time interval $\tau \rightarrow 0$, which is a unphysical limit when we consider an ensemble of spheres with finite dimension at a fixed average density $\rho$. Indeed using statistical physics results, one can estimate

$$
\tau \propto \frac{m}{d\|\boldsymbol{p}\| \rho} \propto \frac{m}{d \sqrt{T} \rho}
$$

where $d$ is the particle diameter; it follows that $\tau$ is finite for finite temperatures. The solution $\boldsymbol{P}(t)$ of the stochastic equation is a Gaussian Ornstein-Uhlenbeck process with parameter $\alpha=\gamma / M$ and the covariance matrix of the observables reads

$$
\begin{aligned}
<\Delta P_{i}(t) \Delta P_{j}(t+\Delta t)> & =\delta_{i j} e^{-\alpha \Delta t} T M\left(1-e^{-2 \alpha t}\right) \\
<\Delta X_{i}(t) \Delta P_{j}(t+\Delta t)> & =\delta_{i j} \frac{T}{\alpha} e^{-\alpha \Delta t}\left(1-e^{-\alpha t}\right)^{2} \\
<\Delta X_{i}(t) \Delta X_{j}(t)> & =\delta_{i j}\left[\frac{2 T}{\gamma}\left(t-\frac{2}{\alpha}\left(1-e^{-\alpha t}\right)\right)+\frac{1}{2 \alpha}\left(1-e^{-2 \alpha t}\right)\right]
\end{aligned}
$$

so that for $t \alpha \gg 1$ we get

$$
<\Delta X_{i}^{2}(t)>\simeq \frac{2 T}{\gamma} t
$$

whereas we have a ballistic behavior for $t \alpha \ll 1$. We remark as the correlationrelaxation time scale $\alpha^{-1}$ changes proportionally to the mass, so that for a test particle for $M \gg m$ we estimate a correlation time and a relaxation time much longer than for the gas particles. The stationary distribution for the momentum is Gaussian whereas for a gas confined in a finite volume, the spatial density is constant. The kinetic energy $E$ of the particles is distributed according to the Maxwell-Boltzmann distribution $p(E) \propto \exp (-E / T)$. The equilibrium condition is consistent with the state law of gas $P=\rho T$ if one computes the pressure $P$ on a boundary surface. The finite size effects (a finite mass and dimension for the gas particles) introduce a finite collision time scale $\tau$ so that the parameter $\gamma$ (cfr. Eq. (2)) scales as $\tau^{-1}$ for a fixed mass $m$, which depends on the local density. Assuming a finite dimension for gas particles the average collision frequency is estimated by

$$
\tau^{-1} \propto a<\Delta v>_{+} \rho
$$


where the symbol $\langle\Delta v\rangle_{+}$means that we take the average value only for the positive values of the relative velocity along a given direction and $a$ is an effective surface for collisions. $\langle\Delta v\rangle_{+}$is estimated by $T^{1 / 2}$ so that, in the equilibrium condition, we expect that $\tau$ scales by $T^{1 / 2}$. A finite $\tau$ could prevent the application of the CLT to justify the stochastic equation for the test particle, when the condition $\Delta t \gg \tau$ is not satisfied. But, for the equilibrium state, the homogeneity of particle distribution implies that the distribution of the collision events does not depend from the position and the momentum dynamics is still described by a Gaussian process. This result is also true if the test particle is a gas particle with a finite mass, so that the probability distribution for the coordinates and momentum are representative for the density distribution of the same quantities for the whole gas.

We now consider the problem if a stochastic model could be justified in the case of a non-equilibrium stationary state (NESS): more precisely we assume that the gas particle are in contact with two thermal reservoirs at different temperature so that we have a temperature gradient along the $x$-direction in the system and we focus our analysis of the dynamics along this direction. We simulate the thermal reservoir $T$ by using a reflecting boundary such that, each time a gas particle hits a barrier, it is reflected in a elastic way and the reflected velocity is distributed according to

$$
\rho(v) \propto v \exp \left(-\frac{m v^{2}}{2 T}\right) \quad v \geq 0
$$

which is the distribution of the particles velocity of a 2-dimensional gas at equilibrium temperature $T$. As a matter of fact the particle distribution relaxes to a stationary state after a certain time and the pressure equilibrium at any cross section of the system gives $P=\rho(x) T(x)$ so that

$$
\rho(x) \propto T^{-1}(x)
$$

Assuming a temperature gradient of the form

$$
T(x)=T_{0}+\beta\left(x-x_{0}\right) \quad x \in\left[x_{0}, x_{1}\right]
$$

where the parameter $\beta=\left(T_{1}-T_{0}\right) /\left(x_{1}-x_{0}\right)$ defines the gradient, the stationary condition implies

$$
\rho(x) \propto \frac{1}{T_{0}+\beta\left(x-x_{0}\right)}
$$

so that the particle density increases near the cold barrier $T_{0}$. In the 2-dimensional case one can estimate the mean free path of the particles $\lambda$ as the probability to collide with a particle in a given volume

$$
\lambda(x) \propto \rho^{-1}(x)
$$

Then if the density is small, two successive collisions of the gas particle may involve particles with different temperatures. In such a case the local thermalization of a 
particle is not justified (i.e. the position $x$ and the momentum $p$ cannot be considered constant when one considers successive collisions) and we have to take into account a correlation effect in the collision dynamics. In such a case the stochastic differential equation (3) has to be modified and the Stratonovich interpretation has to be applied since the effect of a successive collision depends on the previous one. For a test gas particle in a NESS state we apply the following stochastic equation

$$
\begin{aligned}
& d p_{x}=-\frac{\gamma(x)}{m} p_{x} d t+\sqrt{2 \gamma(x) T\left(x+\frac{p_{x}}{\gamma(x)}\right)} d w^{*}(t) \\
& d x=\frac{p_{x}}{m} d t
\end{aligned}
$$

where $d w^{*}$ is the Wiener differential in the Stratonovich interpretation. We remark that $m / \gamma$ is a characteristic correlation time for the collision dynamics of the ensemble particles and that we preserve the Einstein condition. However, the temperature of the colliding particles is not constant when one considers successive collisions since the mean free path is not small. Recalling the definition (2) and the scaling law for the collision time $\tau \propto \sqrt{T}$, we derive the functional form for the drift coefficient

$$
\gamma(x)=\frac{\hat{\gamma} m}{\sqrt{T(x)}}
$$

If one estimates the average momentum evolution in Eq. (9) we have two contributions: the $x$-dependence of the drift term gives

$$
-\left\langle\frac{\gamma(x)}{m} p_{x}\right\rangle \simeq \frac{1}{2} \frac{\hat{\gamma}}{T^{3 / 2}} \frac{d T}{d x} \frac{T m}{\gamma(x)} \simeq \frac{1}{2} \frac{d T}{d x}
$$

where we compute $<p_{x} x>$ using the relations (5), whereas the fluctuating term according to the Stratonovich interpretation gives

$$
-\frac{\gamma}{2} \frac{\partial}{\partial p} T\left(x+\frac{p}{\gamma(x)}\right)=-\frac{1}{2} \frac{d T}{d x}
$$

We remark as the two terms cancel so that no effective drift can be observed for a gas test particle in the NESS state as expected from the equilibrium condition of pressure. Therefore the Stratonovich interpretation of the stochastic differential equation (9) seems physically justified to describe the evolution of the gas particle in a NESS state. This effect is the consequence of the non local character of the collision interactions when we have finite dimension gas particles and the temperature gradient introduces an effective force that is considered by the Stratonovich interpretation of the equation (9). 

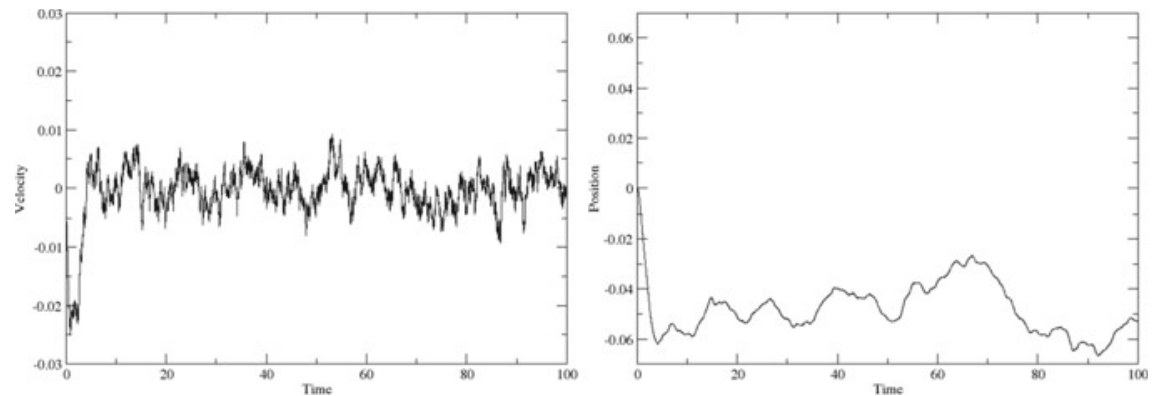

Fig. 1 Numerical solutions of Eq. (9) using the parameters $\hat{\gamma}=1, \beta=.1, T_{0}=.5$ and $T_{1}=2.5$. We have simulated $10^{5}$ particles with $m=1$ in a box $x \in[-10,10]$ with initial condition $x=0, p=0$. The units are arbitrary. The left picture shows the evolution of the average velocity and the right picture shows the evolution of the average position
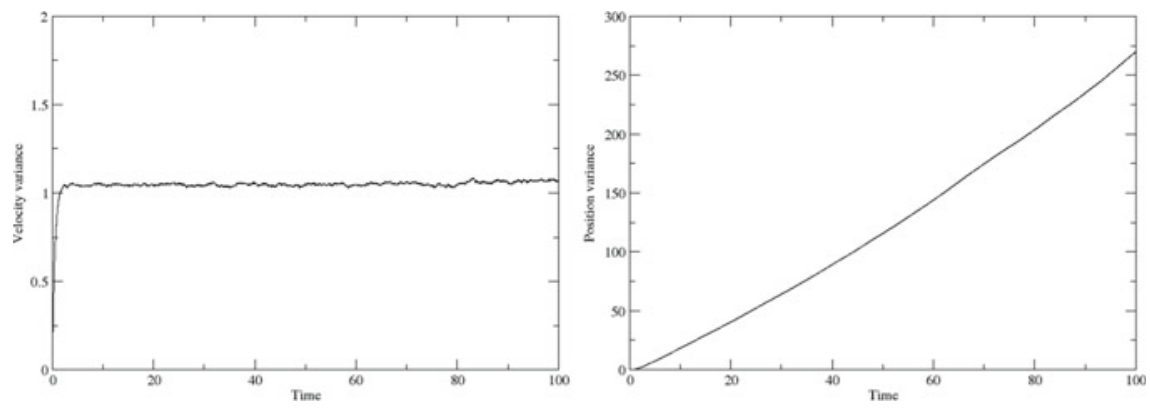

Fig. 2 Numerical solutions of Eq. (9) using the same parameters as in Fig. 1. The left picture shows the evolution of the velocity variance and the right picture shows the evolution of the position variance. We observe a normal diffusion behavior after a short transition time

In Fig. 1 we show the results of a direct integration of Eq. (9) in the Stratonovich interpretation for an ensemble of $10^{5}$ particles (the parameters of the simulation are reported in the caption). We remark the presence of an initial transient regime where we have a drift toward the cold barrier, but then at the stationary state, we get a diffusion regime without drift as it is shown in Fig. 2, where we plot the velocity and position variances. In the case of velocity we reach a stationary value, whereas for the position we get the typical behavior of a local diffusion process: the momentum variance relaxes to the local thermal equilibrium $<\Delta v_{x}^{2}>\rightarrow T / m=1.05$ (according to the parameters values used in the simulations) and the position variance increases linearly with time after a short ballistic regime.

We study as the evolution changes if we consider a test particle of a larger mass $M \geq m$. From a formal point of view equation (9) is modified according to 

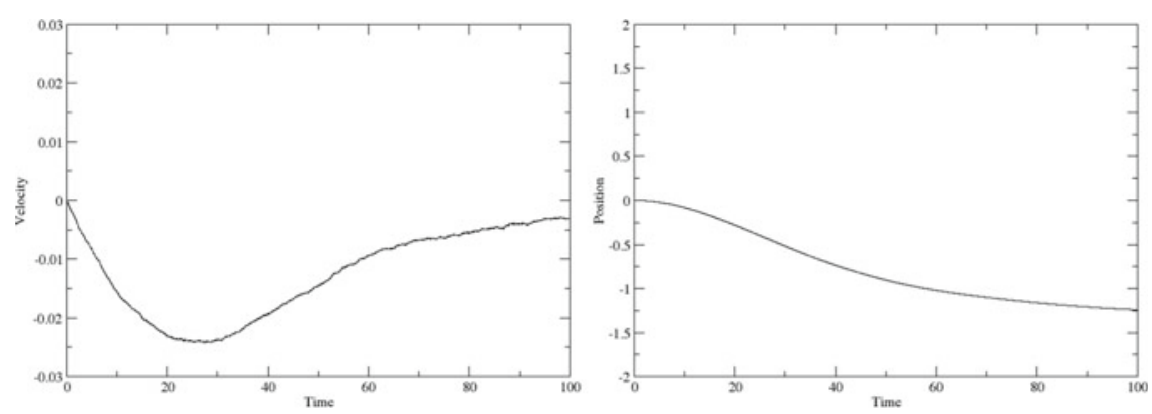

Fig. 3 Numerical solutions of Eq. (10) with $M=25 \mathrm{~m}$, whereas the other parameters are the same as in Fig. 1. The left picture shows the evolution of the average velocity and the right picture shows the evolution of the average position. We observe as the long time correlation induces a drift of the average position toward the negative cold region

$$
\begin{aligned}
& d P_{x}=-\frac{\gamma(x)}{M} P_{x} d t+\sqrt{2 \gamma(x) T\left(x+\frac{P_{x}}{\gamma(x)}\right)} d w^{*}(t) \\
& d X=\frac{P_{x}}{M} d t
\end{aligned}
$$

where the correlation time $\simeq M / \gamma$ is longer than for the gas particles. In such a case the transient regime to reach a stationary condition for the test particle $M$ requires a much longer time and, in the Stratonovich interpretation, we expect a net average displacement toward the cold barrier in the transient regime. We have performed numerical integration of the stochastic equation (10) with $M=25 \mathrm{~m}$ whereas the other parameters are left constant. In Fig. 3 we report the numerical results for the average value of the velocity and the position for an ensemble of test particles. We observe that during long transition time the average velocity takes negative values and we have a net average displacement of the position toward the negative values (i.e. the colder region of the space). We have also checked the variance evolution of the velocity and the position (see Fig. 3). The velocity variance of the massive particles reaches a thermal equilibrium that corresponds to a local temperature associated to the stationary value of the average position, whereas the position variance shows a ballistic behavior for a long time. According to the previous interpretation of the collision dynamics in a thermal bath with a temperature gradient, the existence of a long relaxation time scale implies that a massive particle tends to drift in the colder region simulating the effect of a drift force, until a local equilibrium is reach and the evolution of the particle distribution recall the thermophoresis phenomenon. The overall effect of an ensemble of massive particles starting at a given position is that the distribution moves towards the cold region as shown in Figs. 3 and 4. 
Fig. 4 Empirical distribution function computed using the numerical solution of Eq. (10) after $t=50$ time units. We have used the same parameters as in Fig. 2. We remark that the distribution mode is shifted toward the negative values

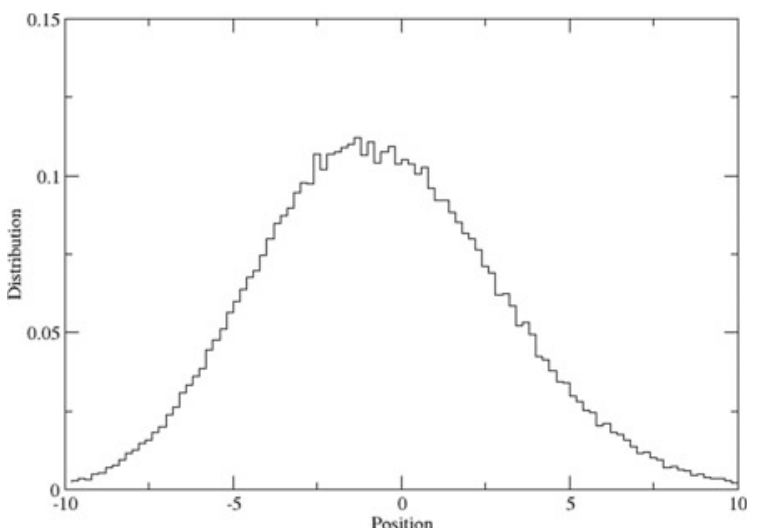

\section{Molecular Dynamics Simulations}

To check the applicability of a stochastic dynamics approach to describe the collision dynamics of a test particle in a thermal bath, we developed a simulation code [9] to perform the molecular dynamics of a 2-dimensional gas composed by elastic spheres that collides between two reflecting horizontal boundary conditions, whereas we have a periodic boundary condition on the vertical axis. The numerical integration performs a event based code algorithm that computes exactly the elastic collision between two rigid spheres and moves the spheres according to a uniform rectilinear motion between two collisions. The event based algorithm [9] is quite efficient and it allows to simulate a great number of particles. The model considers an a fixed dimensional area in the simulations (i.e. a unit square) so that by varying the dimension of the spheres we change the gas particles density. In the sequel we show the simulations using $10^{4}$ rigid spheres of radius $10^{-2}$ that corresponds to an average density $\rho \simeq 3 \%$ defining the mean path length between successive collisions. The gas particle mass is fixed at $m=1$ and the temperature (i.e. the kinetic energy) can be directly related to the evolution time scale so that only the ratio between the chosen values is relevant. We have first checked that in a uniform thermal bath the stochastic differential equation is suitable to describe the statistical properties of the collision dynamics: for a fixed temperature we have checked that the velocity distribution of the gas particle follows a Gaussian distribution (i.e. we get a Maxwell-Boltzmann distribution for the energy) and that the collision time scale $\tau$, which enters in the definition of the drift parameter (see Eq. (2)) scales $\propto \sqrt{T}$. The simulation results are reported in Fig. 5 where we show as in stationary condition the gas particles relax to a thermodynamics equilibrium characterized by the Gaussian distribution: this result is consistent with the results on the dynamical properties of the hard sphere gas studied by the Ergodic Theory [12]. The probability distribution of the collision time $\tau$ is well approximated by an exponential distribution and we observe that the scaling law $\tau \propto \sqrt{T}$ is confirmed by the simulation results. The homogeneity of the 



Fig. 5 Molecular dynamics simulations of a 2-dimensional hard sphere gas in the unit square $(x, y) \in[0,1] \times[0,1]$ using $10^{4}$ particles with $m=1$ and radius $r=.001$ in a thermal bath with temperature $T=1$ (arbitrary unit). In the left picture we show the comparison of the kinetic energy distribution and the Maxwell-Boltzmann distribution (squares) in semilog scale. In the right picture we plot the distribution of the time of flight between successive collisions: the distribution is exponential with a characteristic collision time scale proportional to $T^{1 / 2}$ (see inset in the figure)
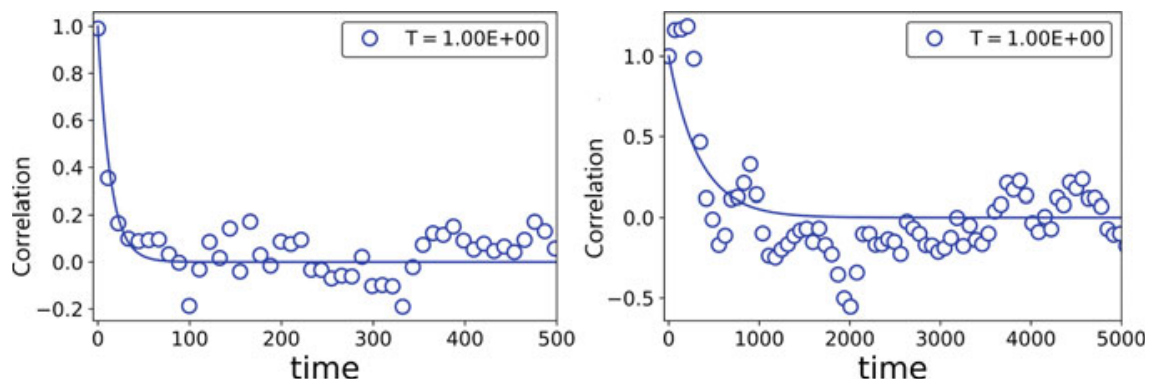

Fig. 6 Time correlation among the trajectories of the hard spheres in the molecular dynamic simulations. The left picture refers to the trajectories of the gas particles $(m=1)$, whereas the right picture refers to the trajectories of the 100 massive test particle $(M=25)$. The time unit is arbitrary

equilibrium state allows to apply the CLT even if the collision time is not negligible with respect to the evolution time scale.

We also checked that the correlation time scale depends from the inverse of the particle mass and the particles diffusion in the system is well described by the stochastic dynamics (3). In Fig. 6 we show the linear correlation in the velocity of the gas particle $(m=1)$ and an ensemble of 100 massive test particles $(M=25)$ in the thermal bath and the increase of the correlation time with the mass is observed. The computation of the time dependence of the position variance both for the gas and the test particles confirms that all the particles perform a normal diffusion with a ballistic transition time and the diffusion coefficient in the stationary regime is independent from the mass as implied by the Einstein relation (see Eq. (5)).

Then we introduced a temperature gradient in the system by mean of two reflecting boundary conditions at $x=0$ and $x=1$ that reproduce the velocity distribution (6) at a temperature $T_{0}=.01$ and $T_{1}=.1$ (the unit is arbitrary). We have checked that the density distribution of the gas particles in the NESS state is consistent with the 



Fig. 7 Molecular dynamics simulations of a 2-dimensional hard spheres gas in the presence of a temperature gradient $T \in[.1,1]$. The left picture shows the local temperature (i.e. the kinetic energy) computed dividing the system into 50 slices as a function of the position; the continuous line defines the linear dependence provided by the Statistical Mechanics Theory. The right picture is a snapshot of the molecular dynamic simulations that shows the particle distribution in presence of the temperature gradient
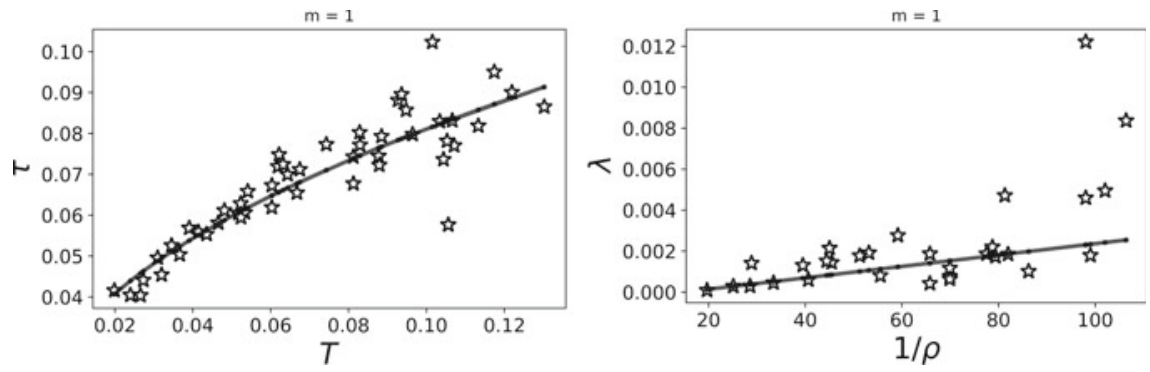

Fig. 8 (Left picture) Empirical dependence of the collision time scale from the local temperature in the hard sphere gas simulations; the continuous curve refers to the interpolation result with $\tau \propto \sqrt{T}$. (Right picture) Mean free path dependence from the inverse of the density; the continuous line is a linear interpolation as provided by the Statistical Mechanics Theory

thermodynamic equilibrium of pressure (7): this is illustrated by the numerical results shown in Fig. 7, where we have divided the system into 50 slices and computed both the local density and temperature of the gas particles. The stochastic differential equation (9) assumes a dependence of the collision time $\tau \propto \sqrt{T}$ that introduces a position dependence of the drift coefficient when a temperature gradient is present (see Fig. 8 left). We have also checked that the mean free path is proportional to the inverse of the local density so that it scales proportionally to the local temperature (see Figs. 7 and 8 right). Finally we introduced 100 test massive particles in the central position $x=.5$ and we have computed the evolution of the average position to check if there can be observed a drift toward the cold barrier during the relaxation process in the NESS state. The numerical results shown in Fig. 9 do not allow to conclude that the test particle dynamics is described by the stochastic differential equation (9) but there is a local negative drift that seems to be statistically relevant. 


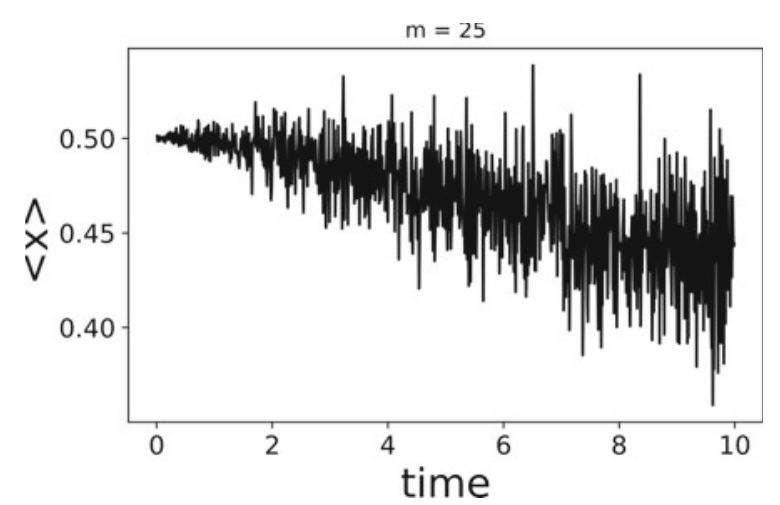

Fig. 9 Evolution of the average position for the ensemble of 100 massive test particles introduced in the hard sphere gas with a temperature gradient $T \in[.1,1]$. The initial position is at $x=.5$. The simulations suggest the existence of an average drift toward the cold barrier

\section{Conclusions}

The aim of this work is to study how the stochastic dynamical systems can contribute to understand some aspects of the non-equilibrium statistical physics. We have briefly introduced simple concepts of collision theory that justify the use of stochastic differential equations in the simulation of the Brownian motion of a test particle in a thermal bath produced by a gas of elastic particles. We have proposed to extend this approach to study the dynamics of a massive test particle in the thermal bath where a temperature gradient is present. This extension is consistent with a thermodynamics description of the system assuming a local equilibrium condition, only if we interpret the stochastic differential equations according to Stratonovich. The theoretical approach points out as a massive particle has a long transient regime in which a average drift effect toward the colder region is observed. This is interpreted as the consequence of the non-local character of the collisions dynamics when one considers finite dimensional particles. In such a way, we prove that the stochastic model is able to describe a thermophoresis phenomenon. due to the long time correlation in the dynamics without the intervention of an external force. We take advantage from a simulation code able to integrate exactly the hard sphere dynamics of ensemble of $2 \mathrm{~d}$-particles and we have performed a first validation of the assumptions that justify the stochastic model. The simulations show that a thermodynamics approach related to the existence of a local equilibrium is realized even in presence of a temperature gradient. These results could be interesting to understand the problem connected to the NESS formation and the local Entropy production [2]. Moreover the simulations point out the existence an average drift toward the colder barrier for an ensemble of massive particles. Such a phenomenon could be interesting in biochemical reactions where large molecules move in a thermal bath (usually defined by water molecules) and the particles dimension is not negligible when a temperature gradient is present. 


\section{References}

1. Schadschneider, A., Chowdhury, D., Nishinari, K.: Stochastic Transport in Complex Systems, 1st edn. Elsevier Science (2010)

2. Jiang, D.-Q., Qian, M., Qian, M.-P.: Mathematical Theory of Nonequilibrium Steady States. Lecture Notes in Mathematics, Springer, Berlin Heidelberg (2004)

3. Jaynes, E.T.: The Minimum Entropy Production Principle. Ann. Rev. Phys. Chem. 31, 579-601 (1980)

4. Seifer, U.: Stochastic thermodynamics: principles and perspective. Eur. Phys. J. B 64(3-4), 423 (2008)

5. Chena, L.Y.: Nonequilibrium fluctuation-dissipation theorem of Brownian dynamics. J. Chem. Phys. 129(14), 144113 (2008)

6. Moreau, M.: On the derivation of the Onsager relations from the Master equation. Lett. Math. Phys. 1, 715 (1975)

7. Arnold, L.: Stochastic Differential Equations Theory and Applications. Dover Publications (2013)

8. Duhr, S., Braun, D.: Why molecules move along a temperature gradient. PNAS 103(52), 19678$19682(2006)$

9. Monti, M., Montanari, C.E.: Getting started with NOCS. https://githib.com/carlidel/pynocs (2019)

10. Seifert, U.: Stochastic thermodynamics: principles and perspectives. Eur. Phys. J. B 64, 423431 (2008)

11. Landau, L.D., Lifshitz, E.M.: Course of Theretical Physics: Statistical Mechanics. Pergamon Press (1980)

12. Simányi, N.: Ergodicity of hard spheres in a box. Ergodic Theory Dyn. Syst. 19(3), 741-766 (1999) 